\title{
Cardiovascular morbidity in subjects with Obstructive sleep apnea and Its correlation with the severity of disease
}

\author{
Sudip S Sachdev, J C Suri, A K Jain, Anita Khalid, H S Isser, M K Mittal \\ Departments of Internal Medicine, Pulmonary Medicine, Cardiology and Radiology, Safdarjung Hospital \\ and Associated Vardhman Mahavir Medical College, New Delhi
}

Indian J Sleep Med 2009; 4.2, 49-60

\begin{abstract}
There is paucity of Indian data evaluating the relationship of hypertension, ventricular dysfunction and carotid artery intima media thickness with Obstructive sleep apnea hypopnea syndrome (OSAHS) and its severity. This observational study compared these outcomes amongst subjects with and without OSAHS. Subjects categorized as 'High Risk' on Berlin's questionnaire underwent polysomnography. The outcomes were evaluated by history, examination, electrocardiography, echocardiography and carotid artery ultrasonography. There were 48 cases (polysomnography confirmed OSAHS) and 44 controls (without OSAHS). Of the 48 cases, $28(58 \%)$ were classified as severe and $20(42 \%)$ non-severe (mild and moderate). A significant association independent of confounders was documented between OSAHS and: (i) blood pressure and hypertension; (ii) previous myocardial infarction, diastolic dysfunction, left ventricular hypertrophy, pulmonary hypertension and arrhythmias; and (iii) carotid artery plaques and intima-media thickness. However, there was no significant impairment of ejection fraction or systolic dysfunction. No statistically significant "dose response" relationship was evident on comparing subjects with severe and non-severe OSAHS.

Keywords: Carotid intimal media thickness, Hypertension, Obstructive Sleep Apnea Hypopnea Syndrome, Ventricular dysfunction.
\end{abstract}

\section{Introduction}

I

n western populations, Obstructive sleep apnea hypopnea syndrome (OSAHS) is frequently associated with increased cardiovascular morbidity and mortality from both ischemic heart disease (interquartile risk ratio of 1.3) and stroke (1.2). OSAHS is also commonly associated with systemic hypertension independent of age, obesity or

\section{Address for correspondence}

\section{Dr J C Suri}

Senior Chest Physician \& Head-Department of Pulmonary, Critical Care \& Sleep Medicine, Vardhman Mahavir Medical College \& Safdarjung Hospital, New Delhi. E-mail: jcsuri@rediffmail.com other confounding factors (3-5). Nocturnal disturbances of cardiac rhythm have also been reported (2). Epidemiological data also suggest an association with congestive cardiac failure (CCF) (2). About half of a small sample of patients with diastolic dysfunction had an apnea hypopnea index $>10$ (6). Mild daytime pulmonary hypertension is a common complication in patients with OSAHS with a prevalence of approximately $20 \%$ (7). Stroke has been linked to OSAHS in both cross sectional and case control studies and sleep apnea is highly prevalent in patients with stroke $(2,8,9)$.

It is primarily over the past two to three decades that implications of OSAHS on cardiovascular diseases have been recognized from data in developed countries. Despite this, even in the west the general cardiovascular community has 
been slow in assimilating OSAHS into the cardiovascular diagnostic and therapeutic paradigms (2). South Asian countries are currently witnessing a rapid escalation of cardiovascular morbidity including ischemic heart disease and hypertension (10). The role of traditional risk factors in cardiovascular morbidity have been extensively evaluated in our setting including obesity, diet, physical inactivity, smoking, alcohol intake, diabetes, and metabolic syndrome. However, there is a paucity of information regarding the relationship of OSAHS with cardiovascular morbidity in Indian adults. In view of the rapid escalation of cardiovascular morbidity in India, it would be important to evaluate its association with OSAHS. The present study was therefore designed to evaluate cardiovascular morbidity in subjects with OSAHS and to correlate the severity of cardiovascular morbidity with the severity of OSAHS.

\section{Subjects and Methods}

The subjects were screened and recruited from the Medicine Outpatient Department, Respiratory Clinic and Internal Medicine wards of Safdarjung Hospital and associated Vardhman Mahavir Medical College, New Delhi. The Postgraduate thesis committee of the institution had approved the study and an informed written consent was taken from the participants.

The pre-specified inclusion criteria for eligibility for the study were: (i) either gender; (ii) age between 30 to 69 years; and (iii) treatment naïve for OSAHS. Subjects with the following conditions were excluded: (i) life threatening illnesses; (ii) pregnancy; (iii) psychosis; (iv) malignancy; (v) end stage renal disease; and (vi) cirrhosis and fulminant hepatic failure. Screening for obstructive sleep apnea hypopnea syndrome was done with the Berlin's questionnaire (11). Subjects categorized as 'High Risk' on the Berlin's questionnaire were requested to undergo sleep studies by polysomnography. There were 48 cases in which OSAHS was diagnosed by polysomnography and 44 controls that were not diagnosed to have OSAHS by Berlin's questionnaire and/or polysomnography.

A detailed history, especially of snoring, excessive daytime somnolence, nocturia, witnessed apneas, choking, waking up unrefreshed in the morning and personality changes were recorded. A thorough general physical and systemic examination was performed. Anthropometry recordings included: (i) weight in $\mathrm{kg}$ on a spring balance calibrated daily; (ii) height in cm on a stadiometer; (iii) body mass index calculated as weight in $\mathrm{kg}$ divided by height in meters square; (iv) waist and hip circumferences in $\mathrm{cm}$ by fiberglass tape using standard techniques. Mean of three readings of blood pressure were recorded by a calibrated mercury sphygmomanometer in consonance with recommendations (12). Laboratory investigations performed included hemogram, plasma glucose, serum sodium and potassium, lipid profile and arterial blood gas analysis, if required (Eishweiler System).

Sleep studies were performed on Alice 5 Healthdyne Polysomnography System (Respironics, USA). A whole night fully supervised, manually validated, level 1 complete polysomnography was conducted. Sleep staging was performed using the criteria of Rechtshaffen and Kales (13). Apnea was defined as the cessation of airflow for 10 seconds or longer. Hypopnea was defined as a recognizable transient reduction (but not complete cessation) of breathing for 10 seconds or longer, a decrease of greater than $50 \%$ in the amplitude of validated measures of breath reduction or decrease of $<50 \%$ associated with oxygen desaturation of $3 \%$ or more (14). Flow limitation was defined as any series of 2 or more breaths, lasting greater than 10 seconds that have flattened or non-sinusoidal appearance on the inspiratory nasal cannula flow signal and end abruptly with a return to breaths with sinusoidal shape. Desaturation was defined as fall of $>3 \%$ in saturation from the baseline following an obstructive event. The number of such episodes per hour was taken as desaturation index (DI). Respiratory distress index (RDI) was calculated as a sum of apnea, hypopnea and flow limitation. Arousal was defined as EEG alpha bursts exceeding 3 seconds during non rapid eye movement (NREM) sleep and bursts with increased chin muscle tone during rapid eye movement (REM) sleep. All non-respiratory arousals were eliminated. The number of arousals per hour was taken as the arousal index (AI). The minimum arterial blood oxygen saturation attained during sleep was also recorded. Categorization of severity of OSAHS was done according to RDI value into three groups: mild $(\mathrm{RDI}=5-19)$, moderate $(\mathrm{RDI}=20-39)$, and severe $(\mathrm{RDI}$ $\geq 40$ ).

Either overnight (cases) or daytime (controls) electrocardiographic recording was done to document arrythmias. Nighttime recording was done via polysomnographic machine and the daytime recording of ECG with long Lead II.

High resolution ultrasonography by Philips HDI 4000 machine was performed to determine the presence of carotid artery plaques and intima-media thickness, which is considered to be an early marker of progression of 
atherosclerosis. The intima-media thickness (IMT) and lumen diameter measurements were performed 1 to $2 \mathrm{~cm}$ proximal to the carotid bifurcation. The sound beam was adjusted perpendicular to the far wall of the vessel, thereby obtaining two parallel echogenic lines corresponding to lumen intima-media and media-adventitia interfaces. These two parallel line echoes were separated by a small echo free space. The intima-media thickness (IMT) was measured between these two leading edges corresponding to the far wall of the common carotid artery (CCA). At each longitudinal projection, the IMT was measured in three segments of the common carotid artery on each side at the point of greatest thickness and at two points $1.0 \mathrm{~cm}$ upstream and $1.0 \mathrm{~cm}$ downstream from the point of greatest thickness (depending on individual variation in vessel wall, tortuosity and habitus). The mean of 6 IMT measurements ( 3 from left and 3 from right common carotid artery) was used as representative value for each subject $(15,16)$. An artery was classified as being affected by a plaque if there was a localized thickening of $>1.5 \mathrm{~mm}$ that did not uniformly involve the whole left or right carotid artery with or without flow disturbance (17). Echocardiography was performed on (Philips Sonos 5500 USA) machine. Special efforts were made to document the following parameters: ejection fraction, diastolic dysfunction, systolic dysfunction, hypertrophy of ventricles and pulmonary artery pressure.

Data was entered and analyzed in EPI-INFO and SPSS (Version 13) statistical softwares. The primary outcomes of interest in relation to cardiovascular morbidities were hypertension, ventricular dysfunction and carotid artery intima-media thickness. These and other outcome variables were compared amongst subjects with OSAHS and controls and amongst severe and non-severe categories of OSAHS. The relationship of various outcome variables with group (case or control) and severity of OSAHS was analyzed with and without adjustment for confounding factors (age, gender and body mass index). The statistical tests employed included Chi-square test, Fischer exact test, Student ' $t$ ' test, Pearson's correlation coefficient, univariate and multiple regression analysis and univariate and multiple logistic regression analysis. A two-tailed $\mathrm{P}$ value below 0.05 was considered to denote statistical significance.

\section{Results}

Of the 48 cases, 10 (21\%) were classified as mild, 10 (21\%) as moderate and $28(58 \%)$ as severe OSAHS. The disease duration was $1-3$ years in 20 (42\%), 4-6 years in $20(42 \%)$ and above 6 years in $8(16 \%)$ cases.

\section{Comparison of Cases and Controls}

Amongst the compared characteristics (Table 1), the cases were older (7 years; $\mathrm{P}<0.001)$, smoked more often and had less positive family history of hypertension, coronary artery disease and diabetes. Subjects with OSAHS were significantly heavier with a greater body mass index, waist to hip circumference and neck circumference. Cases had significantly lower total serum cholesterol, high-density lipoprotein, and low-density lipoprotein. OSAHS was associated with significantly greater C-Reactive Protein levels and non-outcome morbidities including overweight or obesity, diabetes, dyslipidemia and metabolic syndrome.

In view of significant differences between cases and controls for age and body mass index, further comparative analysis for outcome measures of interest also used adjustment for these variables. Three analytic strategies were utilized for comparisons (Table 2): (i) unadjusted, (ii) adjusted for age and gender, and (iii) additional adjustment for body mass index, a close correlate of OSAHS.

Unadjusted and adjusted (age and sex) systolic and diastolic blood pressures were significantly higher amongst subjects with OSAHS (Table 2). However, on additional adjustment for BMI, the differences were not statistically significant. The risk of being hypertensive was significantly higher in cases (Odds Ratio 6.42; 95\% Confidence Interval 2.59 to 15.92 ; $\mathrm{P}<0.001)$ even after adjustment for age and gender (OR 6.22; 95\% CI 2.24 to 17.28 ; $<<0.001$ ). However, on additional adjustment for BMI, the risk of being hypertensive was not statistically significant $(P=0.15)$. The blood pressure readings had been taken while the subjects were consuming prescribed anti-hypertensive drugs. An additional comparison was therefore done for current hypertension (systolic blood pressure $\geq 140 \mathrm{~mm} \mathrm{Hg}$ or diastolic blood pressure $\geq 90 \mathrm{~mm} \mathrm{Hg}$ ) despite being on antihypertensive medication. None of the controls receiving anti-hypertensive drugs had current hypertension. Thus the odds ratio for hypertension despite medication was not measurable. However, on evaluating the Omnibus tests of model coefficients by introducing adjustment variables in a graded manner (analogous to Fischer exact test), there was a significantly higher risk of being currently hypertensive despite being on anti-hypertensive drugs ( $\geq 1$ or $\geq 2$ ). These differences remained highly significant despite adjustment for age and gender, and also additionally for BMI. Only 
Table 1: Comparison of characteristics amongst cases \& controls

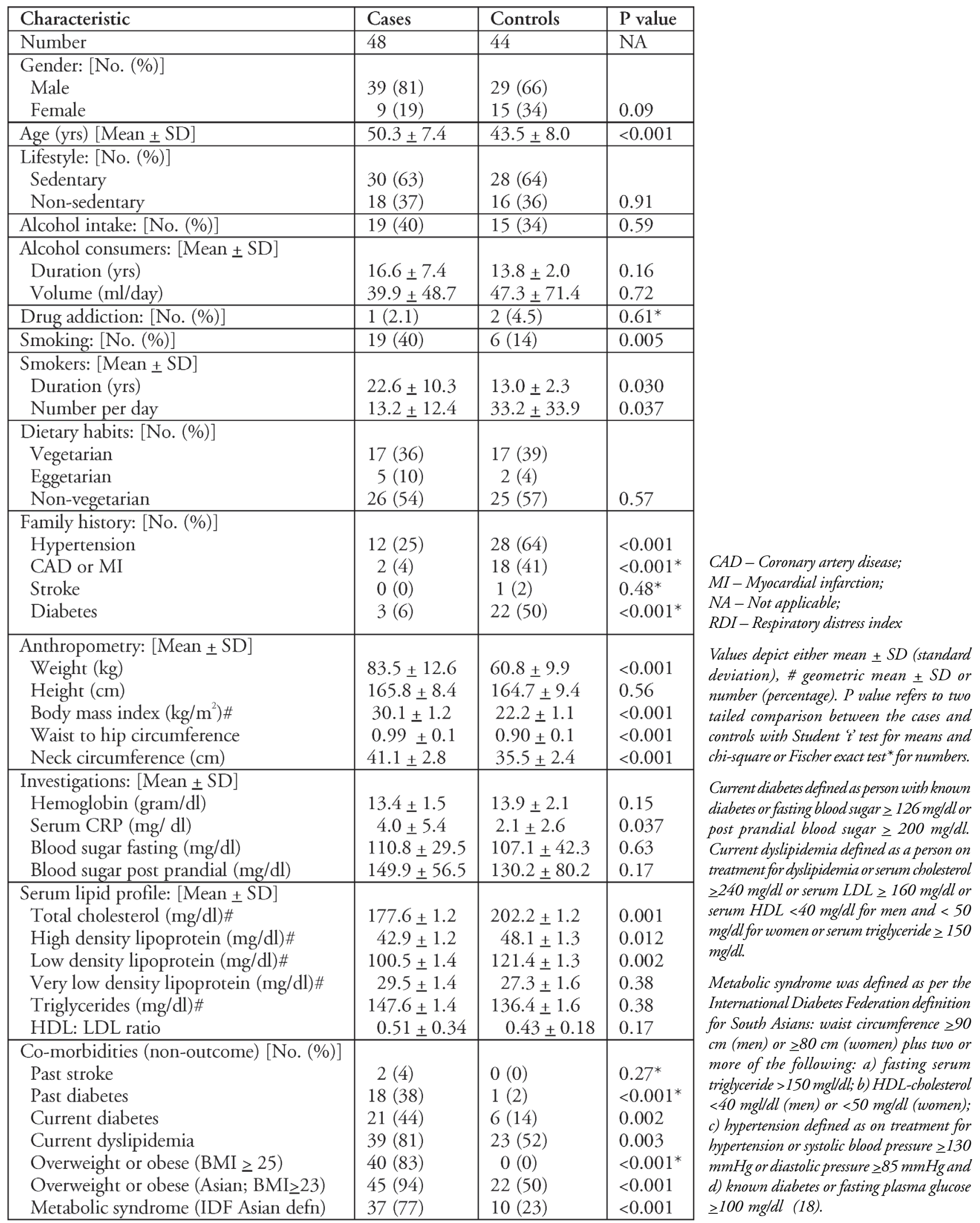


Table 2: Comparison of outcome measures amongst cases and controls

\begin{tabular}{|c|c|c|c|c|}
\hline Characteristic & Controls & Cases & $\begin{array}{l}\text { Mean difference or } \\
\text { OR }(95 \% \mathrm{CI})\end{array}$ & P value \\
\hline Number & 44 & 48 & NA & NA \\
\hline \multicolumn{5}{|l|}{ Blood pressure } \\
\hline $\begin{array}{l}\text { Systolic blood pressure }(\mathrm{mm} \mathrm{Hg})[\mathrm{Mean} \pm \mathrm{SD}] \\
\text { Unadjusted } \\
\text { Adjusted (age, sex) } \\
\text { Adjusted (age, sex, BMI) }\end{array}$ & $\begin{array}{ll}124.6 \quad \pm \\
14.7\end{array}$ & $\begin{array}{l}134.0 \\
\pm 15.5\end{array}$ & $\begin{array}{l}9.3(3.1,15.5) \\
9.8(3.0,16.5) \\
2.2(-7.0,11.3)\end{array}$ & $\begin{array}{l}0.004 \\
0.004 \\
0.006 \\
0.64\end{array}$ \\
\hline $\begin{array}{l}\text { Diastolic blood pressure }(\mathrm{mm} \mathrm{Hg})[\text { Mean } \pm \text { SD] } \\
\text { Unadjusted } \\
\text { Adjusted (age, sex) } \\
\text { Adjusted (age, sex, BMI) }\end{array}$ & $\begin{array}{ll}78.8 & \pm \\
11.9\end{array}$ & $\begin{array}{l}84.7 \pm \\
11.6\end{array}$ & $\begin{array}{l}5.9(1.1,10.7) \\
8.0(2.9,13.1) \\
5.1(-1.9,12.1)\end{array}$ & $\begin{array}{l}0.017 \\
0.017 \\
0.003 \\
0.16\end{array}$ \\
\hline $\begin{array}{l}\text { Hypertensive* [No. (\%)] } \\
\text { Unadjusted } \\
\text { Adjusted (age, sex) } \\
\text { Adjusted (age, sex, BMI) }\end{array}$ & $13(30)$ & $\begin{array}{l}35 \\
(73)\end{array}$ & $\begin{array}{l}6.42(2.59,15.92) \\
6.22(2.24,17.28) \\
2.76(0.69,11.02)\end{array}$ & $\begin{array}{r}<0.001 \\
<0.001 \\
<0.001 \\
0.150\end{array}$ \\
\hline $\begin{array}{l}\text { Current hypertension on } \geq 1 \text { antihypertensive drugs \# [No. } \\
(\%)] \\
\text { Unadjusted } \\
\text { Adjusted (age, sex) } \\
\text { Adjusted (age, sex, BMI) }\end{array}$ & $0(0)$ & $\begin{array}{l}19 \\
(40)\end{array}$ & $\begin{array}{l}\mathrm{NE} \\
\mathrm{NE} \\
\mathrm{NE}\end{array}$ & $\begin{array}{l}<0.001^{* *} \\
<0.001^{* *} \\
<0.001^{* *} \\
<0.001^{* *}\end{array}$ \\
\hline $\begin{array}{l}\text { Current hypertension on } \geq 2 \text { antihypertensive drugs \#\# [No. } \\
(\%)] \\
\text { Unadjusted } \\
\text { Adjusted (age, sex) } \\
\text { Adjusted (age, sex, BMI) }\end{array}$ & $0(0)$ & $\begin{array}{l}12 \\
(25)\end{array}$ & $\begin{array}{l}\mathrm{NE} \\
\mathrm{NE} \\
\mathrm{NE}\end{array}$ & $\begin{array}{l}<0.001^{* *} \\
<0.001^{* *} \\
<0.001^{* *} \\
<0.009^{* *}\end{array}$ \\
\hline $\begin{array}{l}\text { Current hypertension on } 3 \text { antihypertensive drugs \#\# [No. } \\
(\%)]\end{array}$ & $0(0)$ & $2(4)$ & $\mathrm{NE}$ & $0.27^{* *}$ \\
\hline \multicolumn{5}{|l|}{ Echocardiography and Electrocardiography } \\
\hline $\begin{array}{l}\text { Previous myocardial infarction [No. (\%)] } \\
\text { Unadjusted } \\
\text { Adjusted (age, sex) } \\
\text { Adjusted (age, sex, BMI) }\end{array}$ & $0(0)$ & $8(17)$ & $\begin{array}{l}\mathrm{NE} \\
\mathrm{NE} \\
\mathrm{NE}\end{array}$ & $\begin{array}{l}0.006^{* *} \\
0.001^{* *} \\
0.002^{* *} \\
0.006^{* *}\end{array}$ \\
\hline $\begin{array}{l}\text { Ejection fraction (\%) }[\text { Mean } \pm \text { SD }] \\
\text { Unadjusted } \\
\text { Adjusted (age, sex) } \\
\text { Adjusted (age, sex, BMI) }\end{array}$ & $71.9 \pm 5.1$ & $\begin{array}{l}67.4 \pm \\
11.1\end{array}$ & $\begin{array}{l}-4.5(-7.2,-0.9) \\
-3.7(-7.7,0.3) \\
0.2(-5.3,5.6)\end{array}$ & $\begin{array}{l}0.017 \\
0.017 \\
0.072 \\
0.951\end{array}$ \\
\hline $\begin{array}{l}\text { Systolic dysfunction [No. (\%)] } \\
\text { Unadjusted } \\
\text { Adjusted (age, sex) } \\
\text { Adjusted (age, sex, BMI) }\end{array}$ & $0(0)$ & $3(6)$ & $\begin{array}{l}\mathrm{NE} \\
\mathrm{NE} \\
\mathrm{NE}\end{array}$ & $\begin{array}{l}0.24^{* *} \\
0.046^{* *} \\
0.079^{* *} \\
0.59^{* *}\end{array}$ \\
\hline $\begin{array}{l}\text { Diastolic dysfunction [No. (\%)] } \\
\text { Unadjusted } \\
\text { Adjusted (age, sex) } \\
\text { Adjusted (age, sex, BMI) }\end{array}$ & $9(21)$ & $\begin{array}{l}33 \\
(69)\end{array}$ & $\begin{array}{l}8.56(3.30,22.20) \\
6.82(2.43,19.12) \\
3.0(0.73,12.28)\end{array}$ & $\begin{array}{c}<0.001 \\
<0.001 \\
<0.001 \\
0.13\end{array}$ \\
\hline $\begin{array}{l}\text { Pulmonary artery hypertension [No. (\%)] } \\
\text { Unadjusted } \\
\text { Adjusted (age, sex) } \\
\text { Adjusted (age, sex, BMI) }\end{array}$ & $0(0)$ & $\begin{array}{l}10 \\
(21)\end{array}$ & $\begin{array}{l}\mathrm{NE} \\
\mathrm{NE} \\
\mathrm{NE}\end{array}$ & $\begin{array}{l}0.001^{* *} \\
<0.001^{* *} \\
0.003 \\
0.019\end{array}$ \\
\hline $\begin{array}{l}\text { Left ventricular hypertrophy [No. (\%)] } \\
\text { Unadjusted } \\
\text { Adjusted (age, sex) } \\
\text { Adjusted (age, sex, BMI) }\end{array}$ & $5(11)$ & $\begin{array}{l}27 \\
(56)\end{array}$ & $\begin{array}{r}10.03(3.34,29.88) \\
8.48(2.61,27.63) \\
7.49(1.65,33.92)\end{array}$ & $\begin{array}{r}<0.001 \\
<0.001 \\
<0.001 \\
0.009\end{array}$ \\
\hline $\begin{array}{l}\text { Arrhythmias [No. (\%)] } \\
\text { Unadjusted } \\
\text { Adjusted (age, sex) } \\
\text { Adjusted (age, sex, BMI) }\end{array}$ & $0(0)$ & $8(17)$ & $\begin{array}{l}\mathrm{NE} \\
\mathrm{NE} \\
\mathrm{NE}\end{array}$ & $\begin{array}{l}0.006^{* *} \\
0.001^{* *} \\
0.002^{* *} \\
0.006^{* *}\end{array}$ \\
\hline \multicolumn{5}{|l|}{ Carotid artery ultrasound } \\
\hline Previous stroke $[$ No. $(\%)]$ & $0(0)$ & $2(4)$ & $\mathrm{NE}$ & $0.50^{* *}$ \\
\hline $\begin{array}{l}\text { Plaque [No. (\%)] } \\
\text { Unadjusted } \\
\text { Adjusted (age, sex) } \\
\text { Adjusted (age, sex, BMI) }\end{array}$ & $3(7)$ & $\begin{array}{l}13 \\
(27)\end{array}$ & $\begin{array}{l}5.08(1.33,19.27) \\
4.40(0.99,19.52) \\
11.42(1.41,92.87)\end{array}$ & $\begin{array}{l}0.013^{* *} \\
0.017 \\
0.051 \\
0.023\end{array}$ \\
\hline $\begin{array}{l}\text { Intima media thickness }(\mathrm{mm})[\text { Mean } \pm \text { SD }] \\
\text { Unadjusted } \\
\text { Adjusted (age, sex) } \\
\text { Adjusted (age, sex, BMI) }\end{array}$ & $\begin{array}{l}0.85 \\
0.12\end{array}$ & $\begin{array}{l}1.09 \pm \\
0.18\end{array}$ & $\begin{array}{l}0.25(0.18,0.31) \\
0.22(0.15,0.29) \\
0.21(0.11,0.31)\end{array}$ & $\begin{array}{l}<0.001 \\
<0.001 \\
<0.001 \\
<0.001\end{array}$ \\
\hline
\end{tabular}

NA - not applicable; NE - Not estimable

* Defined as physician diagnosed hypertension or on any anti-hypertensive drug medication or systolic blood pressure $\geq 140 \mathrm{~mm} \mathrm{Hg}$ or diastolic blood pressure $\geq 90 \mathrm{~mm} \mathrm{Hg}$.

\# Defined as systolic blood pressure $\geq 140$ $\mathrm{mm} \mathrm{Hg}$ or diastolic blood pressure $\geq 90 \mathrm{~mm}$ $H g$ despite $\geq 1$ anti-hypertensive drugs. \#\# Defined as systolic blood pressure $\geq 140 \mathrm{~mm}$ $\mathrm{Hg}$ or diastolic blood pressure $\geq 90 \mathrm{~mm} \mathrm{Hg}$ despite $\geq 2$ anti-hypertensive drugs. \#\#\# Defined as systolic blood pressure $\geq 140 \mathrm{~mm}$ $\mathrm{Hg}$ or diastolic blood pressure $\geq 90 \mathrm{~mm} \mathrm{Hg}$ despite 3 anti-hypertensive drugs.

** Fischer exact test or Omnibus tests of model coefficients.

None of the case or control subjects had right ventricular hypertrophy.

Amongst arrhythmias 4 subjects had ventricular ectopics, 3 had sinus tachycardia and 1 had sinus bradycardia.

Indian Journal of Sleep Medicine (IJSM), Vol. 4, No. 2, 2009 
two cases with OSAHS had current hypertension despite being on three anti-hypertensive drugs. Thus, subjects with OSAHS had significantly greater severity of hypertension even after adjustment for age, gender and BMI.

Eight cases had a history of previous myocardial infarction in comparison to none, among controls $(\mathrm{P}=0.001$; age and gender adjusted $\mathrm{P}=0.002$; age, gender and $\mathrm{BMI}$ adjusted $\mathrm{P}=0.006)$. The unadjusted ejection fraction was significantly $(\mathrm{P}=0.017)$ lower in subjects with OSAHS (67.4\% vs $71.9 \%$ ) (Table 2$)$. However these differences did not retain significance on adjustment for age and gender, and additionally for BMI. There were no significant differences between cases and controls for systolic dysfunction for the unadjusted and adjusted estimates. The risk of diastolic dysfunction was significantly $(\mathrm{P}<0.001)$ greater in subjects with OSAHS (OR 8.56; 95\% CI 3.3 to 22.2) even after adjustment for age and gender (OR 6.82; $95 \%$ CI 2.43 to $19.12 ; \mathrm{P}<0.001)$. On additional adjustment for BMI the difference was not statistically significant $(\mathrm{P}=0.13)$. Ten cases had pulmonary artery hypertension in comparison to none, among controls $(\mathrm{P}<0.001)$; the statistical significance persisted after adjustment for age and gender $(\mathrm{P}=0.003)$ and additionally for $\mathrm{BMI}(\mathrm{P}=0.019)$. The unadjusted risk for left ventricular hypertrophy was significantly $(\mathrm{P}<0.001)$ greater in subjects with OSAHS (OR 10.03 ; $95 \%$ CI 3.34 to 29.88). This significant risk persisted even after adjustment for age and gender (OR 8.48; 95\% CI 2.61 to 27.63; $\mathrm{P}<0.001)$, and additionally for BMI (OR 7.49; 95\% CI 1.65 to 33.92; $\mathrm{P}=0.009$ ). In comparison to none, among controls eight cases had documented arrhythmias (four had ventricular ectopics, three had sinus tachycardia and 1 had sinus bradycardia) $(\mathrm{P}=0.001)$. This statistical significance persisted after adjustment for age and gender $(\mathrm{P}=0.002)$, and additionally for $\mathrm{BMI}(\mathrm{P}=0.006)$.

There were no significant differences between the two groups for a previous episode of stroke but only two subjects reported this history (Table 2). Subjects with OSAHS had significantly greater carotid artery plaques (OR 5.08; 95\% CI 1.33 to $19.27 ; \mathrm{P}=0.017$ ). The greater risk for plaques was evident even after adjustment for age and gender (OR 4.40; $\mathrm{P}=0.051$ ) and additionally for BMI (OR 11.42; $\mathrm{P}=0.023)$. The unadjusted carotid intima media thickness was significantly greater $(0.25 \mathrm{~mm}, \mathrm{P}<0.001)$ in cases. These significant differences persisted even after adjustment for age and gender $(0.22 \mathrm{~mm} ; \mathrm{P}<0.001)$, and additionally for BMI $(0.21 \mathrm{~mm} ; \mathrm{P}<0.001 \mathrm{~mm})$.

Indian Journal of Sleep Medicine (IJSM), Vol. 4, No. 2, 2009

\section{Comparison of severe and non-severe cases}

The 28 severe cases were compared with the 20 non-severe (mild and moderate) cases. The two groups (non-severe and severe cases) were largely comparable with respect to the various historical, examination and investigative features, and non-outcome co-morbidities (Table 3). The duration of OSAHS was also comparable in the two groups. However, in severe cases neck circumference was significantly $(\mathrm{P}=0.003)$ greater and metabolic syndrome documented more frequently $(\mathrm{P}=0.034)$.

The unadjusted and adjusted estimates of blood pressure, hypertension and current hypertension on antihypertensive drugs were statistically comparable in the two groups, namely, non-severe OSAHS and severe OSAHS (Table 4).

Both groups were comparable with regards to previous history of myocardial infarction (Table 4). The unadjusted and adjusted estimates of various echocardiographic parameters were also statistically comparable between the non-severe and severe cases of OSAHS. These parameters included ejection fraction, systolic and diastolic dysfunction, pulmonary artery hypertension, and left ventricular hypertrophy. Arrhythmias were documented more frequently ( $21 \%$ vs $10 \%)$ in severe cases but the differences were not statistically significant.

Previous stroke and plaques were documented in a comparable proportion of subjects in the two groups (Table 4). The carotid intima media thickness was also statistically comparable amongst subjects with non-severe and severe OSAHS.

\section{Discussion}

The findings from the current study in Indian adults suggest a significant association independent of confounders between obstructive sleep apnea hypopnea syndrome (OSAHS) and the following evaluated cardiovascular morbidities: (i) blood pressure and hypertension; (ii) previous myocardial infarction, diastolic dysfunction, left ventricular hypertrophy, pulmonary hypertension and arrhythmias; and (iii) carotid artery plaques and intimamedia thickness. These data are in conformity with the earlier epidemiologic evidence and suggest that OSAHS is a significant risk factor for cardiovascular morbidity. However, unlike some earlier studies no significant impairment of ejection fraction or systolic dysfunction could 
Table 3 : Comparison of characteristics amongst non-severe and severe OSAHS

\begin{tabular}{|c|c|c|c|}
\hline Characteristic & Non-severe & Severe & P value \\
\hline Number & 20 & 28 & NA \\
\hline \multicolumn{4}{|l|}{ Duration of OSAHS (yrs): [No. (\%)] } \\
\hline $1-3$ & $8(40)$ & $12(43)$ & \\
\hline $4-6$ & $9(45)$ & $11(39)$ & \\
\hline$\geq 7$ & $3(15)$ & $5(18)$ & 0.92 \\
\hline Men: [No. (\%)] & $17(85)$ & $22(79)$ & 0.43 \\
\hline Age (yrs) $[$ Mean \pm SD] & $49.3 \pm 7.2$ & $51.1 \pm 7.6$ & 0.40 \\
\hline Sedentary lifestyle: [No. (\%)] & $13(65)$ & $17(61)$ & 0.76 \\
\hline Alcohol intake: [No. (\%)] & $13(65)$ & $16(57)$ & 0.58 \\
\hline \multicolumn{4}{|l|}{ Alcohol consumers: [Mean + SD] } \\
\hline Duration (yrs) & $17.9 \pm 6.3$ & $15.9 \pm 8.1$ & 0.60 \\
\hline Volume (ml/day) & $52.0 \pm 65.0$ & $32.8 \pm 37.9$ & 0.42 \\
\hline Drug addiction: [No. (\%)] & $1(5)$ & $0(0)$ & $0.42^{*}$ \\
\hline Smoking: [No. (\%)] & $7(35)$ & $12(43)$ & 0.58 \\
\hline \multicolumn{4}{|l|}{ Smokers: [Mean \pm SD] } \\
\hline Duration (yrs) & $22.7 \pm 12.6$ & $22.6 \pm 9.3$ & 0.98 \\
\hline Number per day & $13.7 \pm 9.1$ & $12.9 \pm 14.4$ & 0.90 \\
\hline \multicolumn{4}{|l|}{ Dietary habits: [No. (\%)] } \\
\hline Vegetarian & $9(45)$ & $8(28)$ & \\
\hline Eggetarian & $0(0)$ & $5(18)$ & \\
\hline Non-vegetarian & $11(55)$ & $15(54)$ & $0.11^{*}$ \\
\hline \multicolumn{4}{|l|}{ Family history: [No. (\%)] } \\
\hline Hypertension & $5(25)$ & $7(25)$ & 1.0 \\
\hline CAD or MI & $2(10)$ & $0(0)$ & $0.17^{*}$ \\
\hline Stroke & $0(0)$ & $0(0)$ & NA \\
\hline Diabetes & $1(5)$ & $2(7)$ & $0.63^{*}$ \\
\hline \multicolumn{4}{|l|}{ Anthropometry: [Mean \pm SD] } \\
\hline Weight $(\mathrm{kg})$ & $81.4 \pm 12.8$ & $85.0 \pm 12.4$ & 0.33 \\
\hline Height $(\mathrm{cm})$ & $166.9 \pm 7.5$ & $164.9 \pm 9.0$ & 0.43 \\
\hline Body mass index $\left(\mathrm{kg} / \mathrm{m}^{2}\right) \#$ & $28.9 \pm 1.2$ & $31.0 \pm 1.2$ & 0.18 \\
\hline Waist to hip circumference & $0.99+0.1$ & $1.0+0.1$ & 0.65 \\
\hline Neck circumference $(\mathrm{cm})$ & $39.7 \pm 2.4$ & $42.1+2.7$ & 0.003 \\
\hline \multicolumn{4}{|l|}{ Investigations: [Mean + SD] } \\
\hline Hemoglobin $($ gram/dl) & $13.1 \pm 1.7$ & $13.6 \pm 1.3$ & 0.25 \\
\hline Serum CRP $(\mathrm{mg} / \mathrm{dl})$ & $3.1 \pm 3.7$ & $4.7 \pm 6.3$ & 0.33 \\
\hline Blood sugar fasting $(\mathrm{mg} / \mathrm{dl})$ & $104.8 \pm 25.3$ & $115 . \overline{1} \pm 32.0$ & 0.24 \\
\hline Blood sugar post prandial (mg/dl) & $141.4 \pm 58.7$ & $156.0 \pm 55.2$ & 0.38 \\
\hline \multicolumn{4}{|l|}{ Serum lipid profile: $[$ Mean \pm SD] } \\
\hline Total cholesterol (mg/dl)\# & $181.2 \pm 1.2$ & $175.1 \pm 1.2$ & 0.54 \\
\hline High density lipoprotein (mg/dl)\# & $45.4 \pm 1.2$ & $41.2 \pm 1.2$ & 0.08 \\
\hline Low density lipoprotein $(\mathrm{mg} / \mathrm{dl}) \#$ & $100.3 \pm 1.4$ & $100.7 \pm 1.4$ & 0.97 \\
\hline Very low density lipoprotein $(\mathrm{mg} / \mathrm{dl}) \#$ & $29.7 \pm 1.4$ & $29.4 \pm 1.4$ & 0.94 \\
\hline Triglycerides (mg/dl)\# & $148.3 \pm 1.4$ & $147.1 \pm 1.4$ & 0.94 \\
\hline HDL: LDL ratio & $0.51 \pm 0.32$ & $0.43 \pm 0.16$ & 0.28 \\
\hline \multicolumn{4}{|l|}{ Co-morbidities (non outcome) [No. (\%)] } \\
\hline Past stroke & $1(5)$ & $1(4)$ & $0.27^{*}$ \\
\hline Past diabetes & $6(30)$ & $12(43)$ & 0.36 \\
\hline Current diabetes & $6(30)$ & $15(54)$ & 0.11 \\
\hline Current dyslipidemia & $14(70)$ & $25(89)$ & 0.09 \\
\hline Overweight or obese $(\mathrm{BMI} \geq 25)$ & $16(80)$ & $24(86)$ & 0.60 \\
\hline Overweight or obese (Asian; $\mathrm{BMI} \geq 23$ ) & $18(90)$ & $27(96)$ & 0.36 \\
\hline Metabolic syndrome (IDF Asian defn) & $12(60)$ & $25(89)$ & 0.034 \\
\hline
\end{tabular}

$C A D$ - Coronary artery disease; $M I-$ Myocardial infarction; NA-Not applicable; $R D I-$ Respiratory distress index

Values depict either mean $\pm S D$ (standard deviation), \# geometric mean $\pm S D$ or number (percentage). P value refers to two tailed comparison between the cases and controls with Student 't test for means and chi-square or Fischer exact test ${ }^{*}$ for numbers. Current diabetes defined as person with known diabetes or fasting blood sugar $\geq 126 \mathrm{mg} /$ dl or post prandial blood sugar $\geq 200 \mathrm{mg} / \mathrm{dl}$. Current dyslipidemia defined as a person on treatment for dyslipidemia or serum cholesterol $\geq 240 \mathrm{mg} / \mathrm{dl}$ or serum $L D L \geq 160 \mathrm{mg} / \mathrm{dl}$ or serum $H D L<40$ $\mathrm{mg} / \mathrm{dl}$ for men and $<50 \mathrm{mg} / \mathrm{dl}$ for women or serum triglyceride $\geq 150 \mathrm{mg} / \mathrm{dl}$.

Metabolic syndrome was defined as per the International Diabetes Federation definition for South Asians: waist circumference $\geq 90$ $\mathrm{cm}$ (men) or $\geq 80 \mathrm{~cm}$ (women) plus two or more of the following: a) fasting serum triglyceride $>150 \mathrm{mglldl}$; b) $\mathrm{HDL}$ cholesterol $<40 \mathrm{mgl} / \mathrm{dl}$ (men) or $<50 \mathrm{mg} /$ $d l$ (women); c) hypertension defined as on treatment for hypertension or systolic blood pressure $\geq 130 \mathrm{mmHg}$ or diastolic pressure $\geq 85 \mathrm{mmHg}$ and d) known diabetes or fasting plasma glucose $\geq 100 \mathrm{mg} / \mathrm{dl}$ (18). 
Table 4: Comparison of outcome measures amongst non-severe and severe OSAHS.

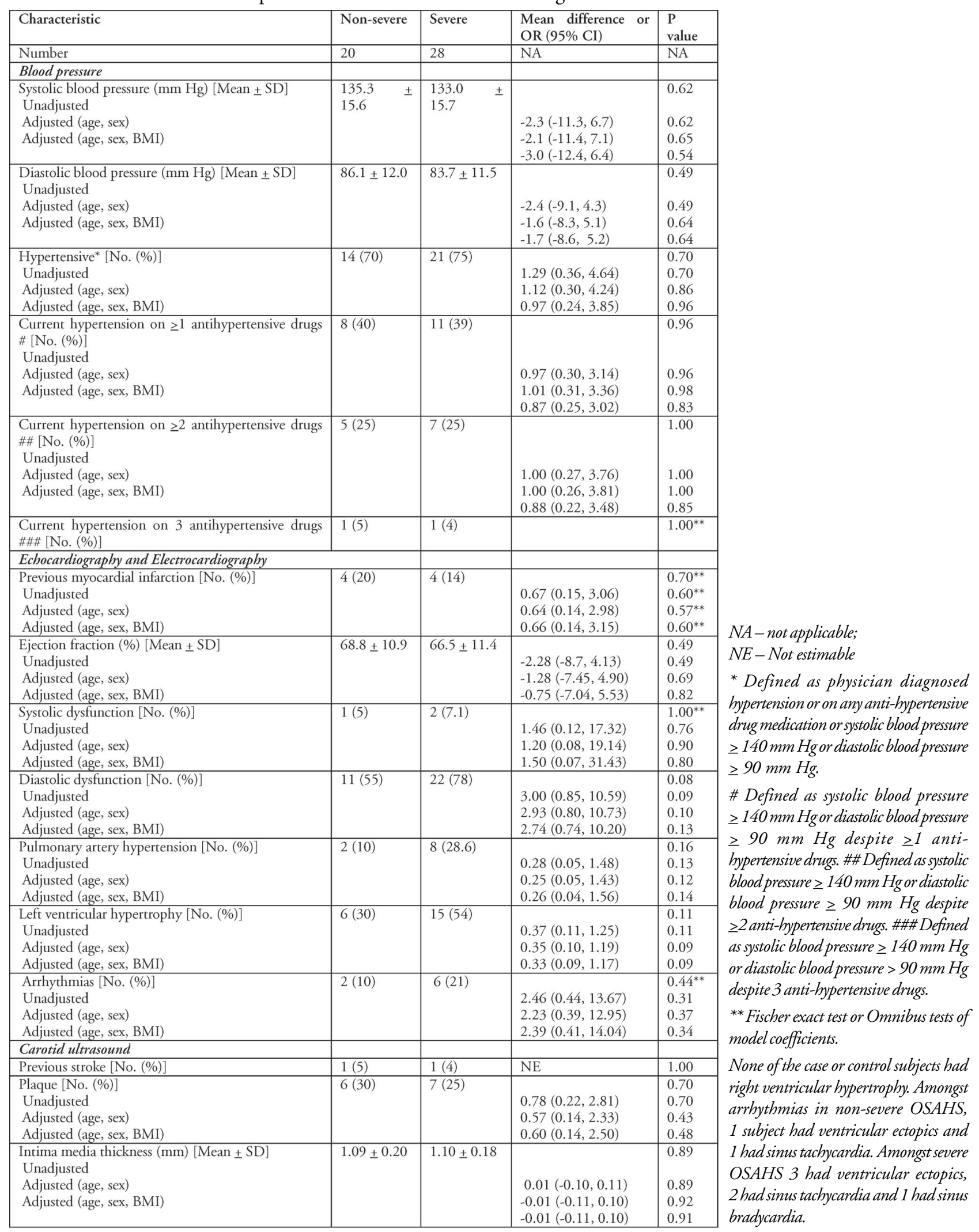

Indian Journal of Sleep Medicine (IJSM), Vol. 4, No. 2, 2009 
be documented probably due to lower sample size. The lack of a statistically significant "dose response" relationship on comparing subjects with severe and non-severe OSAHS is probably a reflection of the smaller sample size and noncomparison of the extreme tails of the disease spectrum.

\section{Comparison of confounders and non- outcome co-morbidities}

Subjects with OSAHS were significantly older, heavier and more obese and had greater associated co-morbidities including diabetes, dyslipidemia and metabolic syndrome. All these variables are well-documented correlates of cardiovascular morbidities including hypertension, ventricular dysfunction and carotid vascular health $(2,19$ 22 ). These variations partially stem from the study design including it's setting in a tertiary care hospital of an urban metro. In any study of this magnitude and nature, it would be virtually impossible to balance all known confounders and co-morbidities across the two arms. Potential controls matched for age and body mass index, either failed to clear the screening criteria of Berlin's questionnaire or declined consent. The older and obese potential controls usually declined consent because of their preoccupation with the primary morbidity necessitating the hospital visit or refusal by younger relatives to accompany them for participation in a study.

The clustering of co-morbidities including obesity, diabetes, dyslipidemia and metabolic syndrome in subjects with OSAHS is not unexpected. There is abundant epidemiological evidence documenting the co-existence of OSAHS and these morbidities $(2,19,20)$. This has led some workers to propose that OSAHS is a manifestation of the metabolic syndrome $(23,24)$. The proposed clinical entity "Syndrome Z" includes the combination of metabolic syndrome and OSAHS $(25,26)$.

A simple comparison of outcomes (cardiovascular morbidities) amongst subjects with and without OSAHS will obviously confuse the interpretation. It was therefore important to adjust for differences by multiple regression analysis with due cognizance to the available sample sizes. Two types of adjustments were utilized: (i) gender and age; (ii) gender, age and BMI. In the latter adjustment, BMI was a good surrogate for most of the co-morbidities (diabetes, dyslipidemia and metabolic syndrome). Addition of all confounders and co-morbidities as adjustment co-variables would not have been feasible with the available sample size and would have caused collinearity with BMI, which was highly correlated with other co-morbidities.

\section{Blood pressure and hypertension}

The unadjusted, age and gender adjusted estimates of systolic and diastolic blood pressure and hypertension were significantly greater in subjects with OSAHS. This is in conformity with earlier evidence from the literature, which is largely from the western world $(2,3,5,19,20)$. However, on additional adjustment for BMI, these differences did not retain statistical significance. This observation is at variance with the earlier data, which documented a significantly greater risk of elevated blood pressure and hypertension after adjustment for several potential confounders including BMI $(2,3,5,19,20)$. The possible explanations for this are: (i) The available sample size did not have adequate power to detect such differences as the variation increased with additional adjustment. The documented odds ratio of 2.76 after adjustment for BMI is within the reported adjusted range (1.37 to 2.89$)$ from the literature $(2,19,20)$; however the $95 \%$ confidence intervals are relatively wide ( 0.69 to 11.02$)$ indicating wider variability. (ii) The blood pressure readings were recorded and hypertension was defined with subjects on anti-hypertensive drugs. As a greater proportion of the cases were consuming anti-hypertensive drugs (34/48 or $71 \%$ vs $2 / 44$ or $5 \%$; $\mathrm{P}<0.001$ ), there was a bias for lowering the actual differences amongst the two groups, which could result in false negative statistical significance; (iii) BMI may represent the common pathway in subjects with OSAHS for elevation of blood pressure; for example it has been proposed that OSAHS may be added as a feature of metabolic syndrome (23-26) and obesity is an essential component of this malady. Control for BMI could thus conceivably represent an over adjustment leading to loss of statistical significance; and (iv) The remote possibility of misclassification of subjects with mild OSAHS as controls could not be excluded with certainty.

Nevertheless, even after adjustment for BMI (good surrogate for other confounders), the risk for severer forms of hypertension was significantly greater in subjects with OSAHS. This is in conformity with earlier evidence $(2,19,20)$.

On comparing subjects with severe and non severe OSAHS, no significant differences were observed for the unadjusted and adjusted estimates of blood pressure, hypertension and current hypertension on anti-hypertensive medication. This finding is at variance with some earlier evidence, which indicates a "dose response" relationship $(2,19,20)$. The possible reasons for not demonstrating statistical significance in the current study could be the relatively small sample sizes for comparison in the two groups

Indian Journal of Sleep Medicine (IJSM), Vol. 4, No. 2, 2009 
(28 and 20 subjects in the two arms). Post-hoc power calculations from the current study suggest that 150 subjects each would be required with severe and non-severe OSAHS to detect with $80 \%$ power and $95 \%$ confidence: (i) a difference of $5 \mathrm{~mm}$ in systolic blood pressure; or (ii) an odds ratio of 2 for hypertension. Also the contrast did not relate to the extreme ends of the spectrum of severe and mild disease.

\section{Echocardiography, Ventricular dysfunction and Arrhythmias}

There was a significant $(\mathrm{P} \leq 0.006)$ association of unadjusted and adjusted (including BMI) estimates of previous myocardial infarction in subjects with OSAHS. This observation is in conformity with the earlier literature documenting an increased cardiovascular morbidity and mortality from ischemic heart disease $(1,2,20)$.

The unadjusted ejection fraction was significantly lower (4.5\%; $\mathrm{P}=0.017$ ) amongst subjects with OSAHS. However these differences did not retain statistical significance on adjustment for age, sex and additionally for BMI. There were no statistical differences for the unadjusted and adjusted estimates for systolic dysfunction; this condition was however documented infrequently (3 cases versus no controls). These findings are slightly at variance with earlier studies, which documented an increased risk of heart failure also after adjustment for several potential confounders $(1,2,19,20)$. This is probably a reflection of the available sample size.

There was a significantly greater association of OSAHS with diastolic dysfunction and left ventricular hypertrophy for the unadjusted, age and gender adjusted, and age, gender and BMI adjusted (for left ventricular hypertrophy only) estimates. Earlier studies have documented that chronically OSAHS is associated with hypertension and increased left ventricular wall thickness, which may lead to diastolic dysfunction $(27,28)$ and it's reversibility with therapy $(27,28)$. On adding systolic blood pressure and drug intake for hypertension in the regression model, systolic blood pressure emerged a significant predictor of left ventricular hypertrophy (OR 1.06, 95\% CI 1.02 to 1.11 ; $\mathrm{P}=0.008$ ), suggesting its contribution to the observed left ventricular hypertrophy.

Subjects with OSAHS had a significantly greater prevalence of pulmonary artery hypertension even after adjustment for age, gender and BMI. Mild pulmonary artery hypertension is a common complication in patients with OSAHS with a prevalence of approximately 20\% (7).

There was a significantly greater association of OSAHS with arrhythmias even for BMI adjusted estimates $(\mathrm{P}=0.006)$. This finding is in conformity with earlier studies which also documented an increased confounder adjusted risk of arrhythmias, particularly atrial fibrillation and ventricular ectopics $(2,19,20,29,30)$. However some observational trials could not document an increased risk of bradyarrhythmias $(29,31)$.

On comparing subjects with severe and non-severe OSAHS, no significant differences were observed for the unadjusted and adjusted estimates of previous myocardial infarction, ejection fraction, systolic and diastolic dysfunction, pulmonary artery hypertension, left ventricular hypertrophy and arrhythmias. This finding is at variance with some earlier evidence, which indicates a "dose response" relationship $(2,19,20,27,28)$. This could be due to relatively small sample sizes and non-comparison of the extreme tails of the spectrum.

\section{Carotid artery ultrasound}

Subjects with OSAHS had a significantly greater risk of carotid artery plaques even after adjustment for age, gender and BMI. This observation is in conformity with the earlier literature documenting an increased occurrence of athersosclerotic plaques in the carotid arteries of OSAHS patients, independent of hypertension $(32,33)$. In the current study also, the greater risk of carotid artery plaques was borderline significant after additional adjustment for hypertension $(\mathrm{P}=0.054)$. However, a few earlier studies with relatively small sample sizes have failed to document a significant increase in the prevalence of plaques in OSAHS $(34,35)$.

The mean carotid intima media thickness was significantly greater in subjects with OSAHS even after adjustment for age, gender and BMI. Earlier studies have documented similar findings without (34-38) and with $(32,33,39,40)$ adjustment for confounders including hypertension. In the current study also, the intima media thickness was significantly greater with additional adjustment for hypertension $(\mathrm{P}<0.001)$.

On comparing subjects with severe and non-severe OSAHS, no significant differences were observed for the unadjusted and adjusted estimates of any of the above evaluated carotid ultrasound parameters. However, a significant association of severe OSAHS with carotid intimal thickness has been reported in unadjusted $(34,37,38)$ and 
adjusted $(35,39,40)$ analyses. The lack of a statistically significant "dose response" relationship is probably a reflection of the smaller sample size and non-comparison of the extreme tails of the spectrum.

In summary, the findings from the current study in Indian adults are in conformity with the earlier epidemiologic evidence and suggest that OSAHS is a significant risk factor for cardiovascular morbidity.

\section{Implications for practice}

It would be prudent to periodically screen subjects diagnosed as OSAHS for various cardiovascular morbidities through echocardiography and carotid ultrasound, if possible. This will result in early identification and therapy of co-existent cardiovascular morbidities, which may improve the prognosis. Conversely, subjects with cardiovascular morbidities, especially refractory hypertension should be screened through questionnaire and treated for OSAHS (if detected), which may prove helpful in alleviating the primary condition.

\section{Implications for future research}

In the Indian setting, larger epidemiological studies will help to clarify the independent relationship between OSAHS and it's severity with the various cardiovascular morbidities. There is thus a need for performing multi-centric studies to achieve the requisite sample size, and also for initiating intervention trials.

\section{References}

1. Shahar E, Whitney CW, Redline S, Lee ET, Newman AB. Sleep disordered breathing and cardiovascular disease: cross sectional results of the Sleep Heart Health Study. Am J Resp Crit Care Med 2001; 163: 19-25.

2. Shamsuzzaman AS, Gersh BJ, Somers VK. Obstructive sleep apnea: Implications for cardiac and vascular disease. JAMA 2003; 290: 1906-14.

3. Peppard PE, Young T, Palta M, Skatrud J. Prospective study of the association between sleep-disordered breathing and hypertension. N Engl J Med 2000; 342: 1378-84.

4. Haas DC, Foster GL, Nieto FJ, Redline S, Resnick S, Resnick HE, Robbins JA, Young T, Pickering TG. Age-dependent associations between sleep-disordered breathing and hypertension. Circulation 2005; 111: 614-21.

5. Nieto FJ, Young TB, Lind BK, et al. Association of sleep disordered breathing, sleep apnea and hypertension in a large community based study. Sleep Heart Health Study. JAMA 2000; 283: 1829-36.
6. Chan J, Sanderson J, Chan W, et al. Prevalence of sleep disorder breathing in diastolic heart failure. Chest 1997; 111:1488-93.

7. Chaouat A, Weitzenblum E, Krieger J, et al. Pulmonary hemodynamics in the obstructive sleep apnea syndrome: results in 220 consecutive patients. Chest 1996; 109: 3806.

8. Good DC, Henkle JQ, Gelber D, et al. Sleep disorder breathing and poor functional outcome after stroke. Stroke 1996; 27: 252-9.

9. Wessendorf T, Dahm C, Teschler H. Prevalence and clinical importance of sleep apnea in the first night after cerebral infarction. Neurology 2003; 60: 1053.

10. Ghaffar A, Reddy KS, Singhi M. Burden of noncommunicable diseases in South Asia. Br Med J 2004; 328: 807-10.

11. Netzer NC, Stoohs RA, Netzer CM, Clark K, Strohl KP. Using the Berlin questionnaire to identify patients at risk for the sleep apnea syndrome. Ann Intern Med 1999;131:48591

12. Williams JS, Brown SM, Conlin PR. Blood pressure measurement. N Engl J Med 2009; 360: e6.

13. Rechtshaffen A, Kales A. A manual of standardized terminology, techniques and scoring system for sleep stages of human sleep. National Institutes of Health, US Government Printing Office, Washington DC, R8, Publication no. 204.

14. American Academy of Sleep Medicine. Sleep related breathing disorders in adults: Recommendations for syndrome definition and measurement techniques in clinical research. Report of an AASM Task Force on Sleep 1999; 22: 667-89.

15. Mohan V, Ravikumar R, Rani SS, Deep R. Intima media thickness of the carotid artery in South Indian diabetic and non- diabetic subjects : the Chennai Urban Population Study (CUPS). Diabetologia 2000; 43: 494-9.

16. Gerulakos G, Ramaswani G, Veuer MG, Fisher GM, Renton $S$, Nicolaides A. Arterial wall changes in type 2 diabetic subjects. Diabetes Med 1994; 11:692 - 5 .

17. Baroncini LAV, de Oliveira A, Vidal EA, Franca GJ, Stahlke PSDB, Alessi A, et al. Appropriateness of carotid plaque and intima-media thickness assessment in routine clinical practice. Cardiovascular Ultrasound 2008; 6: 52 doi:10.1186/1476-7120-6-52.

18. International Diabetes Federation. The IDF consensus worldwide definition of the metabolic syndrome. http:// www.idf.org/webdata/docs/Metac_syndrome_def.pdf; accessed Jan 15th 2009.

19. Douglas Bradley T, Floras JS. Obstructive sleep apnea and its cardiovascular consequences. Lancet 2009; 373 : 82-93.

20. McNicholas WT, Bansignore MR and the management committee of EU COST. ACTION B26. Sleep apnea as an independent risk factor for cardiovascular disease. Current evidence, basic mechanisms and research priorities. Eur Respir J 2007; 29: 156-78.

Indian Journal of Sleep Medicine (IJSM), Vol. 4, No. 2, 2009 
21. Chow CK, Lock K, Teo K, Subramanian S, McKee M, Yusuf $S$. Environmental and societal influences acting on cardiovascular risk factors and disease at a population level: a review. Int J Epidemiol. 2009 Mar 4. [Epub ahead of print].

22. Yusuf S, Hawken S, Ounpuu S, Dans T, Avezum A, Lanas $F$, McQueen M, Budai A, Pais P, Varigos J, Lisheng $L_{i}$ INTERHEART Study Investigators. Effect of potentially modifiable risk factors associated with myocardial infarction in 52 countries (the INTERHEART study): case-control study. Lancet. 2004 ; 364: 937-52.

23. Vgontzas AN, Bixler EO, Chrousos GP. Sleep apnea is a manifestation of the metabolic syndrome. Sleep Med Rev 2005; 9: $211-24$

24. Calvin AD, Albuquerque FN, Lopez-Jimenez F, Somers VK. Obstructive Sleep Apnea, Inflammation, and the Metabolic Syndrome. Metab Syndr Relat Disord. 2009 Apr 3. [Epub ahead of print].

25. Wilcox I. "Syndrome $Z^{\prime}$ : The interaction of sleep apnea, vascular risk factors and heart disease. Thorax 1998; 53; 25-8.

26. Sasanabe RK, Banno K, Otake R, Hasegawa K, Usui MM, Shiomi T. Metabolic syndrome in Japanese patients with obstructive sleep apnea syndrome. Hypertens Res 2006; 29:315-22.

27. Parker JD, Brooks D, Kozar LF et al. Acute and chronic effects of airway obstruction on canine left ventricular performance. Am J Respir Crit Care Med 1999; 160: 188896.

28. Arias MA, Garcia-Rio F, Mediano O, et al. Obstructive sleep apnea syndrome affects left ventricular diastolic dysfunction: Effects of nasal continuous positive airway pressure in men. Circulation 2005; 112: 375-83.

29. Mehra R, Benjamin EJ, Shahar E, et al. Association of nocturnal arrhythmias with sleep-disordered breathing: the Sleep Heart Health Study. Am J Respir Crit Care Med 2006; 173: 910-6.

30. Lanfranchi PA, Somers VK, Braghiroli A, et al. Cebtral sleep apnea in left ventricular dysfunction: prevalence and implications for arrhythmic risk. Circulation 2003; 107: 72732.
31. Flemons WW, Remmers JE, Gillis AM. Sleep apnea and cardiac arrhythmias. Is there a relationship? Am Rev Respir Dis 1993; 148: 618-21.

32. Baguet JP, Hammer L, Levy $P$, et al. The severity of oxygen desaturation is predictive of carotid wall thickening and plaque occurrence. Chest 2005; 128: 3407-12.

33. Suzuki T, Nakano H, Maekawa J, et al. Obstructive sleep apnea and carotid-artery intima-media thickness. Sleep 2004; 27:129-33

34. Altin R, Ozdemir H, Mahmułyazicioðlu K, Kart L, Uzun L, Ozer T, Savranlar A, Aydin M. Evaluation of carotid artery wall thickness with high-resolution sonography in obstructive sleep apnea syndrome. J Clin Ultrasound 2005; 33: $80-6$.

35. Drager LF, Bortolotto LA, Lorenzi MC, Figueiredo AC, Krieger EM, Lorenzi-Filho G. Early signs of atherosclerosis in obstructive sleep apnea. Am J Respir Crit Care Med 2005; 172:613-8. Epub 2005 May 18.

36. Silvestrini M, Rizzato B, Placidi F, Baruffaldi R, Bianconi A Diomedi M. Carotid artery wall thickness in patients with obstructive sleep apnea syndrome. Stroke 2002 ; 33: 1782 5.

37. Li C, Zhang XL, Liu H, Wang ZG, Yin KS. Association among plasma interleukin-18 levels, carotid intima- media thickness and severity of obstructive sleep apnea. Chin Med J (Engl) 2009; 122: 24-9.

38. Tanriverdi H, Evrengul H, Kara CO, Kuru O, Tanriverdi S Ozkurt S, Kaftan A, Kilic M. Aortic stiffness, flow-mediated dilatation and carotid intima-media thickness in obstructive sleep apnea: non-invasive indicators of atherosclerosis. Respiration 2006; 73:733-4.

39. Drager LF, Bortolotto LA, Krieger EM, Lorenzi-Filho G Additive effects of obstructive sleep apnea and hypertension on early markers of carotid atherosclerosis. Hypertension 2009; 53: 64-9. Epub 2008 Nov 17.

40. Saletu $\mathbf{M}$, Sauter C, Lalouschek W, Saletu B, Kapfhammer $\mathrm{G}$, Benesch T, Zeitlhofer J. Is excessive daytime sleepiness a predictor of carotid atherosclerosis in sleep apnea? Atherosclerosis 2008; 196: 810-6. Epub 2007 Mar 13. 